\title{
Correction to: Clinical outcomes in patients with diffuse large B cell lymphoma with a partial response to first-line R-CHOP chemotherapy: prognostic value of secondary International Prognostic Index scores and Deauville scores
}

\author{
Hyewon Lee ${ }^{1,12} \cdot$ Yu Ri Kim ${ }^{2}$. Soo-Jeong Kim ${ }^{3} \cdot$ Yong Park ${ }^{4} \cdot$ Hyeon-Seok Eom ${ }^{1}$. Sung Yong Oh ${ }^{5} \cdot$ Hyo Jung Kim $^{6}$. \\ Hye Jin Kang ${ }^{7} \cdot$ Won-Sik Lee ${ }^{8}$. Joon Ho Moon ${ }^{9} \cdot$ Young-Woong Won ${ }^{10} \cdot$ Tae-Sung Kim ${ }^{11} \cdot$ Jin Seok Kim ${ }^{3}$
}

Published online: 16 December 2019

(C) Springer-Verlag GmbH Germany, part of Springer Nature 2019

\section{Correction to: Annals of Hematology}

https://doi.org/10.1007/s00277-017-3107-6

An additional affiliation for the first author was not indicated. Hyewon Lee is also affiliated with: Department of Internal Medicine, Yonsei University College of Medicine, Seoul, South Korea.

Publisher's note Springer Nature remains neutral with regard to jurisdictional claims in published maps and institutional affiliations.

The online version of the original article can be found at https://doi.org/ $10.1007 / \mathrm{s} 00277-017-3107-6$

Jin Seok Kim

hemakim@yuhs.ac

1 Center for Hematologic Malignancy, National Cancer Center, Goyang, South Korea

2 Department of Internal Medicine, Yonsei University College of Medicine, Gangnam Severance Hospital, Seoul, South Korea

3 Division of Hematology, Department of Internal Medicine, Yonsei University College of Medicine, Severance Hospital, 50-1 Yonsei-ro, Seoul, Seodaemun-gu 03722, South Korea

4 Division of Hematology-Oncology, Department of Internal Medicine, Korea University College of Medicine, Seoul, South Korea

5 Department of Internal Medicine, Dong-A University College of Medicine, Busan, South Korea

6 Division of Hematology-Oncology, Department of Internal Medicine, College of Medicine, Hallym University, Hallym University Sacred Heart Hospital, Anyang, South Korea
Division of Hematology-Oncology, Department of Internal Medicine, Korea Cancer Center Hospital, Korea Institute of Radiological and Medical Sciences, Seoul, South Korea

8 Division of Hematology-Oncology, Department of Internal Medicine, Busan Paik Hospital, Inje University College of Medicine, Busan, South Korea

9 Department of Hematology and Medical Oncology, Kyungpook National University Hospital, Daegu, South Korea

10 Department of Internal Medicine, Hanyang University College of Medicine, Seoul, South Korea

11 Department of Nuclear Medicine, National Cancer Center, Goyang, South Korea

12 Department of Internal Medicine, Yonsei University College of Medicine, Seoul, South Korea 